\title{
Controllable Shrinking and Shaping of Glass Nanocapillaries under Electron Irradiation
}

\author{
L. J. Steinbock, ${ }^{\dagger}$ J. F. Steinbock, ${ }^{\ddagger}$ and A. Radenovic ${ }^{\dagger}{ }^{\dagger}$ \\ ${ }^{\dagger}$ Laboratory of Nanoscale Biology, Institute of Bioengineering, School of Engineering, EPFL, 1015 Lausanne, Switzerland \\ ${ }^{\ddagger}$ Faculty of Physics, Technical University of Kaiserslautern, 67663 Kaiserslautern, Germany
}

\section{Supporting Information}

ABSTRACT: The ability to reshape nanopores and observe their shrinkage under an electron microscope is a powerful and novel technique. It increases the sensitivity of the resistive pulse sensing and enables to detect very short and small molecules. However, this has not yet been shown for glass nanocapillaries. In contrast to their solid-state nanopore counterparts, nanocapillaries are cheap, easily fabricated and in the production do not necessitate clean room facilities. We show for the first time that quartz nanocapillaries can be shrunken under a scanning electron microscope beam. Since the shrinking is caused by the thermal heating of the electrons, increasing the beam current increases the

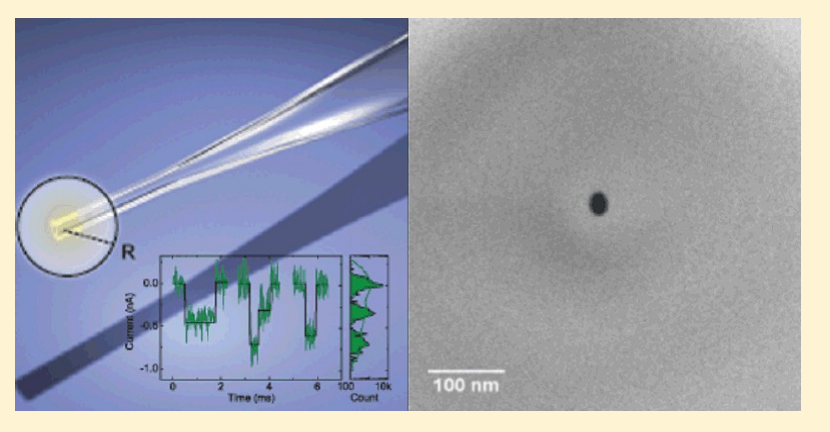
shrink rate. Higher acceleration voltage on the contrary increases the electron penetration depth and reduces the electron density causing slower shrinkage. This allows us to fine control the shrink rate and to stop the shrinking process at any desired diameter. We show that a shrunken nanocapillary detects DNA translocation with six times higher signal amplitudes than an unmodified nanocapillary. This will open a new path to detect small and short molecules such as proteins or RNA with nanocapillaries.

KEYWORDS: Nanocapillary, quartz glass, SEM, shrinking, nanopore, resistive pulse technique

$\mathrm{T}$ he resistive pulse technique (also called Coulter counter technique) is a versatile method to count and characterize single cells, colloids, or even sequence DNA. ${ }^{1-3}$ An electric potential is applied through a small orifice incorporated into a membrane. This potential creates an ionic current whose amplitude depends, besides parameters such as ionic concentration and surface charge, on the volume and shape of the orifice. ${ }^{4}$ Orifices with diameters in the nanometer range are named nanopores, which are divided into solid-state and biological nanopores. ${ }^{5}$

Besides solid-state nanopores in silicon dioxide, silicon nitride, or metal membranes, glass nanocapillaries have emerged in recent years as a cost-effective and versatile source of nanopores for single molecule detection. ${ }^{6}$ They are fabricated using a laser pipettete puller, which heats the cylindrical hollow capillary and stretches it at the same time. This causes the glass capillary to shrink in diameter at the heated spot and finally break into two conical tips defined as nanocapillaries. Depending on the parameters used during the fabrication such as pull strength or heat different end diameters can be reached ranging from micro- to tens of nanometers. ${ }^{7,8}$ This wide range of end diameters, a fast, cheap, and userfriendly fabrication process, and no need for clean rooms or TEMs is an advantage compared to the fabrication needs for other solid-state nanopores. Reliably diameters of $30 \mathrm{~nm}$ can be reached using laser pullers; however to increase the sensitivity for smaller molecules smaller diameters would be essential. ${ }^{9}$
Hence, a technique would be needed to further decrease the diameter of the laser-pulled glass nanocapillaries.

Classical solid-state nanopores have seen a large variety of different techniques emerging in the past decade to decrease their size. Smaller diameters are favorable since they permit to detect smaller molecules because the current change due to the analyte entering the nanopore increases with smaller nanopores. ${ }^{10}$ First experiments were performed by Storm et al. who shrunk nanopores in silicon oxide from 40 to about $3 \mathrm{~nm}$ in diameter using a TEM beam. ${ }^{11}$ The shrinking was explained by the surface stress causing the fluidized silicon oxide to contract in order to minimize the surface of the nanopore. This was followed by experiments where scanning electron microscopes (SEM) were used to either shrink nanopores by silicon oxide deposition ${ }^{12}$ or thermal induced shrinking. ${ }^{13,14}$ The importance of thermal heating was recently demonstrated by Asghar et al. who caused nanopores to decrease their diameter by heating them up in an oven to about $1000{ }^{\circ} \mathrm{C} .{ }^{15}$ Since carbon deposition is often observed under SEM irradiation nanopore shrinking was also investigated with energy-dispersive X-ray spectroscopy (EDX) by Prabhu et al. who could rule out significant carbon deposition. ${ }^{16,17}$ The shrink rates were also examined as a function of the SEM magnification showing a

Received: January 24, 2013

Revised: February 23, 2013

Published: March 18, 2013 

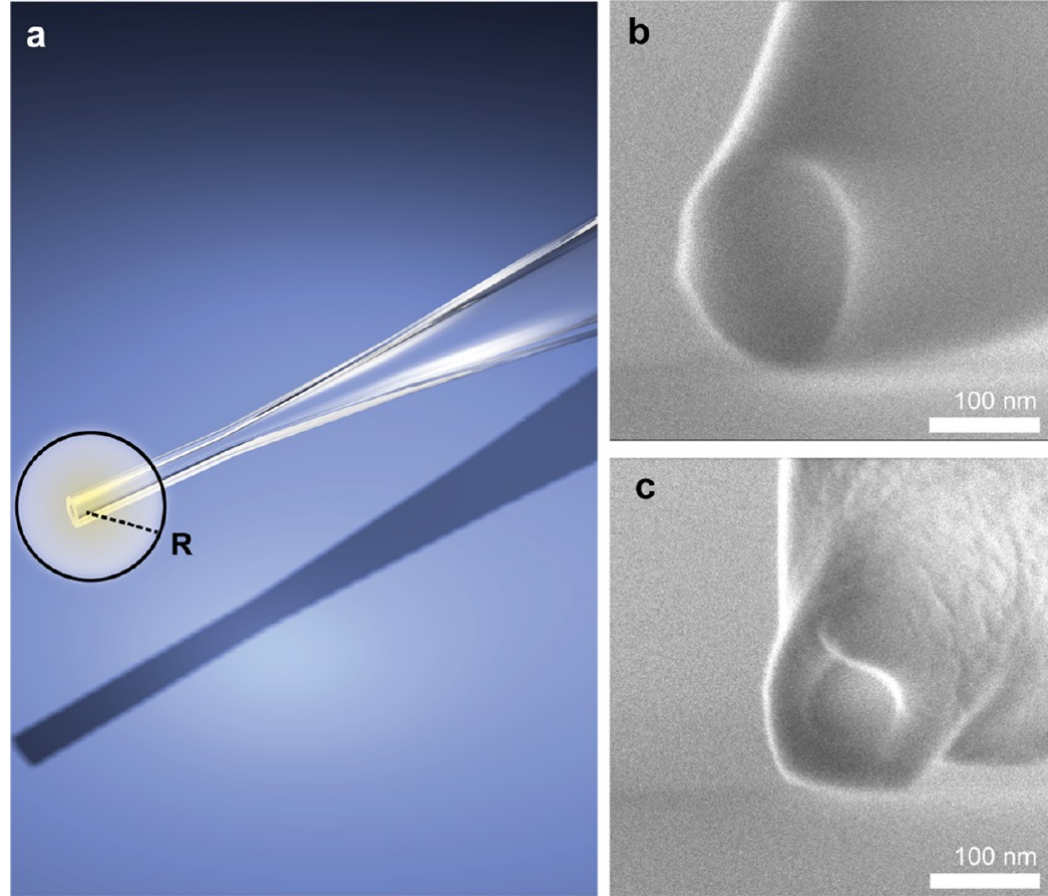

Figure 1. (a) Scheme of the conical end of the nanocapillary. The shaded area depicts the region, which is imaged by the SEM beam. The radius $R$ is the penetration depth of the electron beam calculated by the theoretical penetration formula given by Kanaya-Okayama. (b) SEM in-lens image of a quartz nanocapillary magnified 196k times at a stage angle of 60 degrees to increase the three-dimensional perception. The electron high tension was at $3.0 \mathrm{kV}$, the beam current was at $171 \mathrm{pA}$, and the working distance was $3.3 \mathrm{~mm}$. (c) Shrunken nanocapillary after 14 min of irradiation under constant angle and beam parameters. The process of deformation is also shown in a video in the SI (SI_movie_1.avi). Clearly a reshaping of the nanocapillary is visible, precluding the possibility of significant carbon deposition. This was supported by EDX measurements included in the Supporting Information (Figure S1, Figure S2, and Table S1).

positive linear response to the magnification. ${ }^{16}$ In contrast a power law dependence was observed for the beam potential (also called extra-high tension, EHT), where smaller shrink rates were observed at higher voltages. ${ }^{16,18}$ This was supported by Monte Carlo simulations showing increased penetration depths for electrons at higher beam potentials. The higher penetration depths cause smaller energy densities and hence less thermal heating leading to smaller shrink rates. Both shrinking techniques with a TEM or SEM have the advantage to allow live observation of the shrinking process. This permits to stop the shrinking process at any desired size. A new approach was pursued by Ayub et al. who electrodeposited $\mathrm{Pt}$ on the nanopore interface while already immersed in the ionic solution. ${ }^{19}$ The electrodeposition process is monitored in situ and can be stopped at any desired conductance level. Another technique is atomic layer deposition (ALD), which deposits angstrom thick layers of i.e. aluminum oxide to decrease the nanopore size. ${ }^{20,21}$ Compared to electronic microscopy shrinking, the above-mentioned methods do not permit to see the size of the nanopore but to deduce the diameter from the conductance.

Glass nanocapillaries fabricated directly from a laser pipette puller have not yet benefited from a shrinking method with live optical monitoring of the shrinking nanocapillary. Quartz nanopore membrane (QNM) require the incorporation of a sharp polished Pt wire into a glass membrane, which is etched away while monitoring the conductance of the opening nanopore. ${ }^{22}$ A similar technique is used by Gao et al. who corrode a nanopore into a sealed capillary pipette using an HF solution. ${ }^{9}$ Again the pore opening is controlled nonoptically by measuring the conductance. A novel technique were the pore diameter can even be changed in situ was presented by Platt, Willmott, and Lee who use a tunable pore made out of thermoplastic polyurethane membrane. ${ }^{23}$

We demonstrate a method to shrink quartz glass nanocapillaries to any size from up to $200 \mathrm{~nm}$ to a few nanometers or even to the complete closure of the nanopore. The shrinking occurs while observing the glass nanocapillary under an SEM allowing precise control of their size by terminating the process at any desired diameter. The shrinking is fast reaching $0.25 \mathrm{~nm} /$ $\mathrm{s}$ and can be changed by adjusting the beam current or the electron acceleration voltage. This method has various applications like in the resistive pulse technique, where small diameters increase the sensing sensitivity. This is due to the increase in the amplitude of the current drop, when the molecule enters the nanopore. In the future this will facilitate the detection of very small and short polymers. ${ }^{24}$ The increase in the current amplitude will be demonstrated showing an increase from about $50 \mathrm{pA}$ for single DNA strand for an unmodified nanocapillary to over $300 \mathrm{pA}$ for a shrunken nanocapillary. Other applications which will benefit from a smaller diameter of the nanocapillary include near-field scanning optical microscopy (NSOM), scanning electrochemical microscopy (SECM), or nanobilayer coated glass capillaries. $^{25-27}$

The quartz capillaries were purchased with an inner and outer diameter of 0.3 and $0.5 \mathrm{~mm}$ (Hilgenberg, Germany). The capillary were pulled with the laser pipette puller P-2000 (Sutter, USA). The pulling parameters were Heat 550, Filament 0 , Velocity 50, Deletion 130, and Pull 150 resulting in a single pull after an activated laser for about $1.05 \mathrm{~s}$. This resulted in nanocapillaries with a taper length of approximately $4 \mathrm{~mm}$. A 

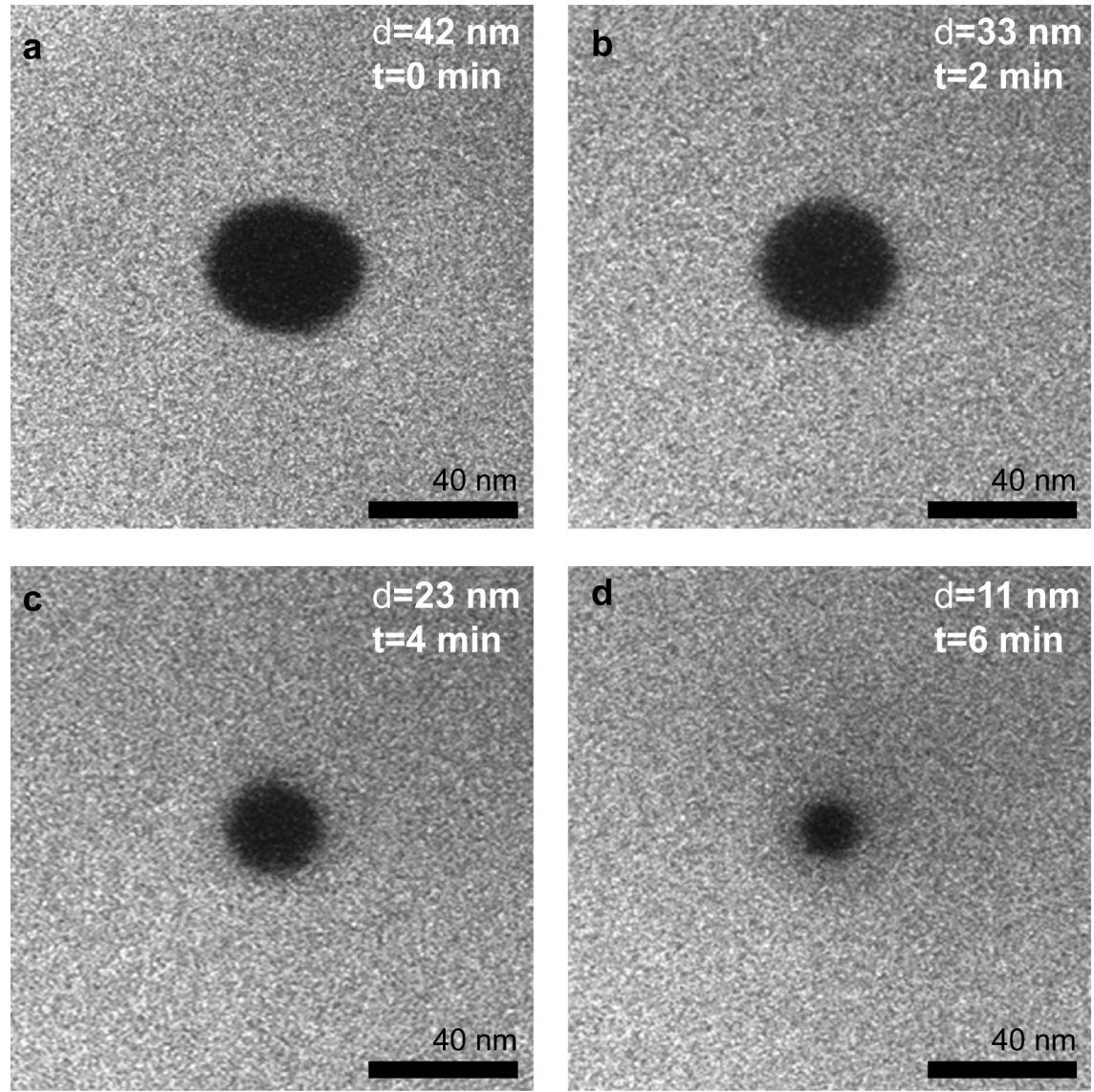

Figure 2. (a) Top side SEM in-lens image of a nanocapillary before the shrinking process at time $t=0$. The beam parameters were hold constant at an electron high tension of $4 \mathrm{kV}$, beam current of $119 \mathrm{pA}$, a magnification of 200k times, and a working distance of $5.1 \mathrm{~mm}$. The analysis of the black hole in the middle with ImageJ resulted in a diameter (d) of $42 \mathrm{~nm}$. (b) After two minutes of constant imaging under the SEM beam first contraction of the diameter can be observed. The analysis of the black pore resulted in an inner diameter of $33 \mathrm{~nm}$. (c) Past 4 min after starting to image the nanopore had further shrunken reaching a diameter of $23 \mathrm{~nm}$. (d) A final diameter of $11 \mathrm{~nm}$ was reached after 6 min. The beam parameters were held constant over the whole shrinking process. Note that the process of shrinking is uniform from all sides. The video of the decreasing diameter can be seen in the SI (SI_movie_2).

detailed description of capillary pulling can be found in previous publications. 6,28

The resulting nanocapillaries from the pull were imaged under a field emission scanning electron microscope (FESEM or SEM). The Merlin SEM (Zeiss, Germany) did not necessitate the presence of a conducting layer on the glass nanocapillaries when imaging with the in-lens detector. This allowed determining the diameter of every nanocapillary before assembling it into the measuring cell, which was not possible before. ${ }^{6}$ SEM imaging was performed under a working distance between 2 and $9 \mathrm{~mm}$, magnifications between 100k and 250k, beam currents between 10 and $800 \mathrm{pA}$, and acceleration voltages of 1-9 kV. EDX measurements were also possible, permitting it to measure the chemical composition before and after the shrinking. EDX measurements were performed using the AZtexEnergy software under a working distance of about 8 $\mathrm{mm}$ and beam potentials of $3 \mathrm{kV}$ or higher.

The nanocapillaries were assembled into a PDMS cell, whose two reservoirs were only connected by the glass orifice. ${ }^{4}$ The bottom of the PDMS cell sealed with a $0.15 \mathrm{~mm}$ thick cover glass (Menzel-Glässer, Germany). The reservoirs were filled with a potassium chloride $(\mathrm{KCl})$ solution of $1 \mathrm{~mol} / \mathrm{L}(\mathrm{M}), 1$ $\mathrm{mM}$ Tris, and $0.1 \mathrm{mM}$ ethylenediaminetetraacetic acid (EDTA) buffer at $\mathrm{pH}$. The solution was cleared from contaminating particles using an anotop 25 filter (Watman, USA). To remove air bubbles inside the nanocapillary after addition of the buffer solution, the PDMS cell was degassed inside a desiccator using a vacuum line. ${ }^{28}$ Oxygen plasma for minutes did improve this step by rendering the surface hydrophilic.

To apply a potential and measure the ionic current, the current amplifier Axopatch 200B was used (Axon Instruments, USA) with a low pass Bessel filter at $10 \mathrm{kHz}$ and a PXI-4461 DAQ card (National Instruments, USA) sampling at a frequency of $100 \mathrm{kHz}$. The electrodes were made out of chlorinated silver electrodes $(\mathrm{Ag} / \mathrm{AgCl})$ which were placed on both sides of the nanocapillary to measure the ionic current through the nanocapillary. The DNA translocation events were recorded and analyzed using a custom written LabVIEW program and a CUSUM algorithm, respectively. ${ }^{29,30}$

We found that the diameter of nanocapillaries made out of quartz glass shrinks similar to silicon nanopores when imaged under SEM electron beam. ${ }^{16}$ This enables one to reach any desired diameter with nanocapillaries. This has an important impact on many fields such as an increased sensitivity for the resistance pulse technique or on the resolution of the scanning electrochemical microscopy. ${ }^{10,25}$

Figure 1a presents a schematic representation of nanocapillary showing the conical shape and the region of the tip, which contains a single nanopore. The shaded circle depicts the area, which is irradiated by the electron beam when imaged by 
the SEM. The penetration depth of the electron entering the quartz glass made out of mainly $\mathrm{SiO}_{2}$ can be estimated by the Kanaya-Okayama depth penetration formula:

$$
R=\frac{0.0276 A U^{1.67}}{Z^{0.89} \rho} \mu \mathrm{m}
$$

The expression describes the penetration depth $R$ of the electrons in dependence from the beam potential $U(\mathrm{kV})$, the atomic weight $(\mathrm{g} / \mathrm{mol}), A$, the atomic number, $Z$, and the density of the imaged material $\left(\mathrm{g} / \mathrm{cm}^{2}\right), \rho$. The penetration depth $R$ is depicted in Figure 1a with a black circle. From this equation one can see, that the penetration depth, $R$, increases with higher beam potentials, $U$. Using the penetration depth an electron density, $d_{\mathrm{e}}$, can be defined as:

$$
d_{\mathrm{e}}=\frac{N_{\mathrm{e}}}{(1 / 2) \pi(R)^{3}(4 / 3)}
$$

$N_{\mathrm{e}}$ represents the number of electrons and $(1 / 2) \pi(R)^{3} 4 / 3$ stands for the hypothetical penetration volume represented by a half sphere. Calculating the electron density $\left(d_{\mathrm{e}}\right)$ once can see that increasing the penetration depth, $R$, by having higher beam potentials decreases $d_{\mathrm{e}}$. Increasing the beam current (number of electrons per time) increases the number of electrons and therefore augments the electron density. If one assumes a linear dependence between the electron density and the energy density, it can be predicted that the energy density and hence the thermal heating will increase with higher beam currents or with smaller beam potentials.

Figure $1 \mathrm{~b}$ shows a side view of a nanocapillary with a vertical inner diameter of about $175 \mathrm{~nm}$ at a working distance of 3.3 $\mathrm{mm}$, a beam current of $171 \mathrm{pA}$ and a beam potential of $3.0 \mathrm{kV}$. While holding the magnification constant at $196 \mathrm{k}$ and imaging the nanocapillary for $14 \mathrm{~min}$ the diameter shrinks approximately to $83 \mathrm{~nm}$ (see Figure 1c). The shrinking of this nanocapillary can be seen in the Supporting Information (SI_movie_1.avi) as a time lapse video. To facilitate determination of the inner diameter nanocapillaries were aligned concentric to the electron beam. This enabled precise determination of its inner diameter (see Figure $2 a-d$ ). The image sequence shows the constant shrinking of the nanocapillary under a beam potential of $4 \mathrm{kV}$, a beam current of 119 pA, a working distance of $5.1 \mathrm{~mm}$, and a magnification of $200 \mathrm{k}$. The nanocapillary at time zero in Figure $2 \mathrm{a}$ has an inner diameter of $42 \mathrm{~nm}$, which constantly shrinks reaching $33 \mathrm{~nm}$ after $2 \mathrm{~min}$ (Figure 2b), $23 \mathrm{~nm}$ after $4 \mathrm{~min}$ (Figure 2c), and 11 $\mathrm{nm}$ after $6 \mathrm{~min}$ (Figure 2d). For kinetics of the process see SI_movie_2.avi in the Supporting Information. Carbon deposition was ruled by EDX measurements shown in the SI (Figures S1 and S2 and Table S1). ${ }^{16}$

To compare the effect of different parameters such as the beam potential or beam current a normalized diameter unit was chosen. For that the diameter value, $D$, was divided at time $\Delta t$ by the initial diameter value, $D_{0}$, at time point zero $(t=0)$. Figure 3a shows the shrinking in the normalized diameter when imaged at different beam potentials ranging from $1.5 \mathrm{kV}$ to 6 $\mathrm{kV}$ and a constant beam current of $119 \mathrm{pA}$. One can see that at lower potentials the shrinking process happens much quicker reaching $25 \%$ of the initial size already after $150 \mathrm{~s}$ at $1.5 \mathrm{kV}$. At higher beam potentials the shrinking process manifests much slower, reaching only $50 \%$ after $600 \mathrm{~s}$ at $6 \mathrm{kV}$. The opposite behavior can be seen when the beam potential was held constant at $3 \mathrm{kV}$, and the beam current was changed. In this
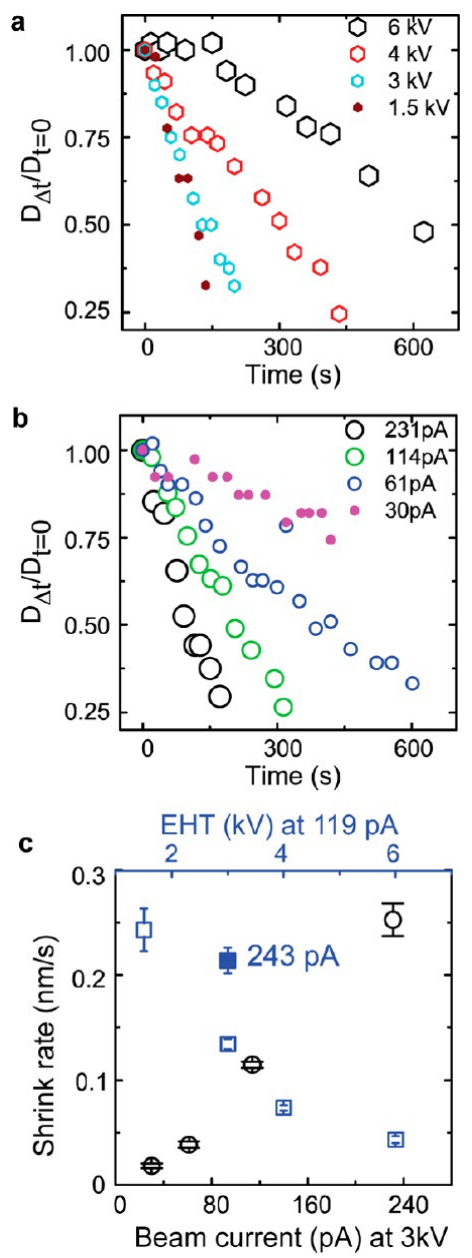

Figure 3. (a) Diameter shrinking over time while irradiated by the SEM beam. The diameter at the different time points was normalized by dividing by the initial diameter at time zero. The shrinking was recorded with a constant beam current of $119 \mathrm{pA}$ but varying beam potential (also called EHT for extra-high tension) between $1.5 \mathrm{kV}$ and $6 \mathrm{kV}$. Interestingly the smaller the electron high tension is, the faster the shrinking of the diameter takes place. While the diameter shrinks to 0.5 of the initial diameter after $150 \mathrm{~s}$ at $1.5 \mathrm{kV}$ it takes over $600 \mathrm{~s}$ to reach this ratio at $6 \mathrm{kV}$. (b) In contrast to part a the beam potential was held constant at $3 \mathrm{kV}$, and the beam current was varied from 30 to $231 \mathrm{pA}$. For a beam current of $231 \mathrm{pA}$ the nanocapillary shrinks to $25 \%$ of its initial size after only $150 \mathrm{~s}$, while a low beam current of 30 $\mathrm{pA}$ it reaches only $75 \%$ of its original diameter after more than $450 \mathrm{~s}$. (c) Shrink rate of the nanocapillary as a function of the beam parameters such as beam current (black lower abscissa) and electron high tension (blue upper abscissa). The shrink rate was calculated from the previous diameter-time dependence in parts a and $b$ by calculating the slope using a linear fit. The shrink rate shows a linear dependence from the beam current (hollow black circles), while a power law becomes apparent for the relation between the shrink rate and the beam potential (hollow blue squares). This is expected from the Kanaya-Okayama formula (eq 1). The filled blue square at beam potential $3 \mathrm{kV}$ was recorded at a beam current of $243 \mathrm{pA}$ twice as high than the other data points at $119 \mathrm{pA}$. The doubled shrink rate is in agreement with the linear dependence between the beam current and the shrink rate.

case lower beam current values induce a slow shrinking and high values and fast shrinking (see Figure $3 \mathrm{~b}$ ). While a beam of 30 pA causes the nanocapillary to shrink to only $75 \%$ after 450 $\mathrm{s}$ the nanopores shrinks to $25 \%$ of its initial size after only $150 \mathrm{~s}$ when imaged at $231 \mathrm{pA}$. Figure $2 \mathrm{a}$ and $\mathrm{b}$ supports our 

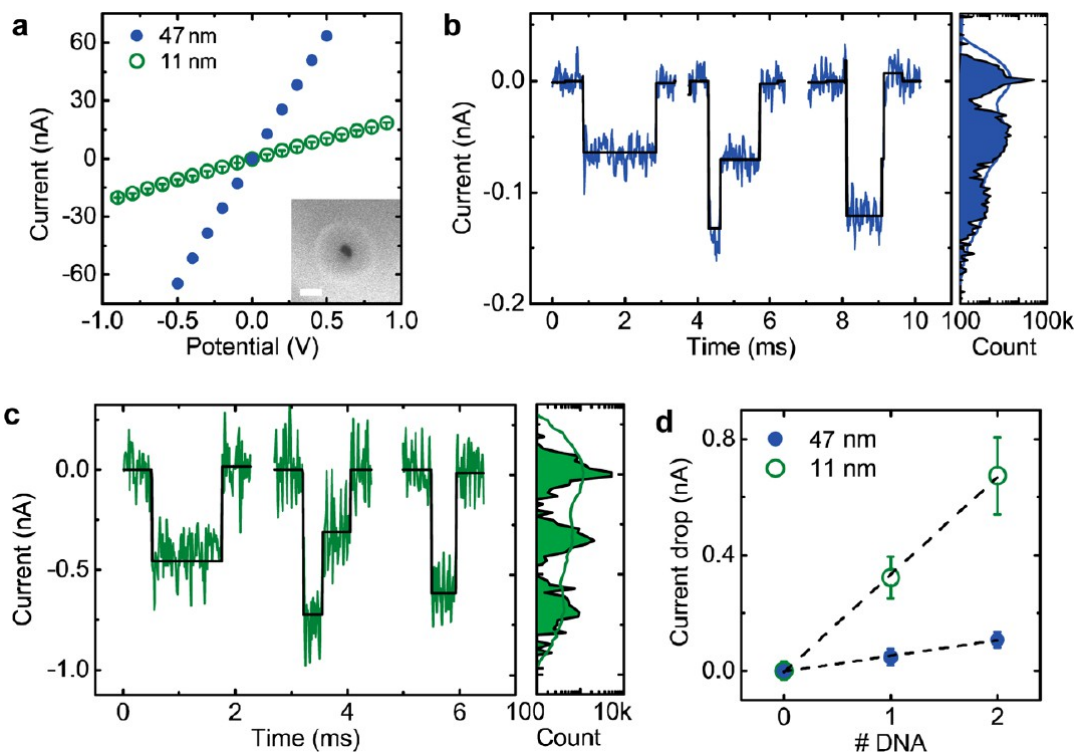

Figure 4. (a) Current in dependence of the applied potential for a nanopore shrunken under an SEM beam to $11 \mathrm{~nm}$ and an unmodified nanopore of approximately $47 \mathrm{~nm}$. The $\mathrm{KCl}$ solution was $1 \mathrm{M}$ for both nanocapillaries and resulted in a conductance of $21 \mathrm{nS}$ for the shrunken nanocapillary and $128 \mathrm{nS}$ for the unmodified nanocapillary. The inset shows the shrunken nanocapillary with a diameter of around $11 \mathrm{~nm}$. The white scale bar represents $20 \mathrm{~nm}$. (b) Current as a function of time showing the translocation of $\lambda$-DNA with three exemplary current drops (blue line) at $0.5 \mathrm{~V}$. The current was normalized to zero to improve comparison between the events. The black line is a CUSUM fit described by Raillon et al. The graph in the right is the histogram of the current traces (blue line) and the fits (black line) for hundreds of events. The histogram shows the quantification of the events with the biggest peak at zero current representing zero DNA strands inside the open nanopore. The blue line is generated from the raw current signal, while the black line results from the CUSUM fit. ${ }^{30}$ The second and third peaks around $49 \mathrm{pA}$ and $109 \mathrm{pA}$ are caused by one and two DNA molecules residing inside the nanocapillary, respectively. (c) Three exemplary current traces (green line) recorded for DNA translocation through the $11 \mathrm{~nm}$ big nanocapillary at $0.5 \mathrm{~V}$. The black line is the result from the CUSUM fit. The right graph is a histogram from several hundreds of events similar to the ones displayed on the left side. The green line is generated from the raw current data, while the black line results from the CUSUM fit. (d) Current drop as a function of the number of DNA strands for the nanocapillary with an inner diameter of $11 \mathrm{~nm}$ (green hollow circles) and $47 \mathrm{~nm}$ (blue circles) when applying $0.5 \mathrm{~V}$. The values were obtained by fitting a Gauss function to the peaks generated from the CUSUM in the histograms of parts $\mathrm{b}$ and $\mathrm{c}$. With current drops of $323 \mathrm{pA}$ for one DNA molecule for the $11 \mathrm{~nm}$ nanocapillary compared to only 49 pA for the $47 \mathrm{~nm}$ nanocapillary a clear increase in the current blockage for smaller nanocapillaries can be observed.

prediction, which anticipates faster shrinking with increasing beam currents but lower shrink rates with increasing beam potentials. To quantify the shrink rate the diameter changes were fitted with a linear function, and the resulting slope was plotted in dependence of the beam current and the beam potential (see Figure 3c). The shrink rate as a function of the beam current shows a linear dependence with a rate ranging from about $0.02 \mathrm{~nm} / \mathrm{s}$ at $30 \mathrm{pA}$ to $0.25 \mathrm{~nm} / \mathrm{s}$ at $240 \mathrm{pA}$ (black circles in Figure $3 \mathrm{c}$ ). In contrast, the shrink rate shows power law dependence when plotted as a function of the beam potential (blue squares in Figure 3c). Both dependences agree with our model. The number of electrons increases linearly with the beam current, augmenting the energy density inside the glass. This causes thermal heating of the nanocapillary and its diameter shrinking due to the surface stress (see eq 2). But when the beam potential is increased the penetration depth of the electron entering the glass is increased. This diminishes the energy density, resulting in less heating and therefore smaller shrink rates with increasing beam potentials (see eqs 1 and 2).

The ability to shrink nanocapillaries to any size has wide applications. One of them is the resistive pulse technique, which profits from a smaller nanocapillary with an increase in the signal amplitude. ${ }^{10,31}$ To prove this a nanocapillary was shrunken to a diameter of $11 \mathrm{~nm}$ and incorporated into a PDMS cell (see SI, Figure 3). The nanocapillary was filled with a $1 \mathrm{M} \mathrm{KCl}$ buffer solution and the current was measured of a range of potentials (see green circles in Figure 4a). Fitting this IV-curve with a linear function reveals the slope which gives a conductance of $21 \mathrm{nS}$. To illustrate the effect of the shrinking an IV-curve was also recorded with an unmodified nanocapillary (blue circles in Figure 4a). The unshrunken nanocapillary with an approximated diameter of $47 \mathrm{~nm}$ showed a higher conductance of $128 \mathrm{nS}$ than the $11 \mathrm{~nm}$ nanocapillary with only $21 \mathrm{nS}$. Next, a $0.5 \mu \mathrm{g} / \mu \mathrm{L} \lambda$-DNA solution of the same ionic strength was added to the reservoir in front of the shrunken and unmodified nanocapillary. A positive potential was applied to the electrode inside the nanocapillary causing the $\lambda$-DNA to translocate into the nanocapillary and reduce the ionic current. ${ }^{32}$ Figure $4 \mathrm{~b}$ and $\mathrm{c}$ shows exemplary current traces recorded at $0.5 \mathrm{~V}$. It is characterized by quantized decreasing steps, revealing the number of DNA strands inside the unmodified (blue trace) and shrunken nanocapillary (green trace). ${ }^{6,33}$ The black line is a fit by a previously described CUSUM algorithm by Raillon et al. The algorithm allows the analysis of noisy data by generating better resolved histograms. ${ }^{30}$ This becomes visible when looking at the histogram in Figure $4 \mathrm{c}$ where the CUSUM generates peaks (black line) which are better pronounced than the peaks from the raw data (green line). The first and biggest peak represents the open pore current and is normalized to zero pA to better compare events in case of changing baseline current. The second and third peaks represent one or two DNA strands inside the nanocapillary. ${ }^{6,33}$ The peaks from the CUSUM fit in the histograms were fitted with Gauss functions to determine their position and plotted in Figure $4 \mathrm{~d}$ against the number of DNA strands. As predicted the current decreases due to DNA strands 
inside the nanocapillary are much bigger when translocating through a smaller nanocapillary than a bigger nanocapillary. While a single DNA strand causes reduction of $49 \mathrm{pA}$ in the unmodified nanocapillary the DNA inside the shrunken nanocapillary (11 nm diameter) generates a more than six time stronger decrease of $323 \mathrm{pA}$. This expected increase in the signal amplitude will permit detecting smaller molecules such as single stranded DNA, RNA or even proteins. Further the statistical analysis of the different folding states shows that unfolded events are much more prominent in the smaller nanocapillary than in the bigger one (Figure S4a and b in the SI) which agrees well with the finding from $\mathrm{Li}$ et al. with silicon nanopores. ${ }^{33}$ Smaller diameter will increase the resolution of scanning electrochemical microscopy or surface near-field optical microscopy, which are both based on conical glass capillaries.

We showed that nanometer-sized orifices in quartz glass can be reshaped using an ordinary scanning electron microscope. The shrinking of the nanocapillary occurs within minutes which allow stopping the process at any desired size ranging from 100 to a few nanometers. The shrinking process was explained with a model based on the penetration depth by Kanaya-Okayama. The model predicts a linear dependence of the shrinking rate from the beam current and a power law dependence for the beam potential. This was shown experimentally for various beam currents and beam potentials. This finding enables to fine-control the shrinking by accelerating or decelerating it, permitting to reach small diameters within seconds or switch to slow and well controlled shrink rate if desired. Interesting avenues to pursue include testing the effect of different pipette shapes and SEM instruments on the shrinking behavior. Further, we have shown that shrinking the inner diameter of nanocapillaries increases the signal amplitude caused by the translocation of DNA. In the future this will make it possible to detect smaller molecules like RNA or proteins translocating through nanocapillaries. Besides improving the resistive pulse technique it will also enhance other techniques like SECM, SNOM, or 3D-printing.

\section{ASSOCIATED CONTENT}

\section{S Supporting Information}

EDX spectra and composition, additional SEM images, SI movie 1.avi, SI movie 2.avi, and DNA folding state statistics. This material is available free of charge via the Internet at http://pubs.acs.org.

\section{AUTHOR INFORMATION}

\section{Corresponding Author}

*E-mail: aleksandra.radenovic@epfl.ch.

\section{Notes}

The authors declare the following competing financial interest(s): The authors have issued a patent on the described process.

\section{ACKNOWLEDGMENTS}

This work and L.J.S. was financially supported by the European Research Council (grant no. 259398, ProABEL: Nanopore integrated nanoelectrodes for biomolecular manipulation and design). Special thanks to Prof. Olivier Martin and Dr. Christian Santschi for providing a pipette puller. SEM and EDX measurements were performed in the EPFL Center for Micro/Nanotechnology (CMI) with technical support from
Zdenek Benes. The authors acknowledge fruitful discussions with F. Traversi and C. Raillon.

\section{REFERENCES}

(1) Coulter, W. H. Means for counting particles suspended in a fluid. U.S. Patent 2656508, 1953.

(2) Steinbock, L. J.; Stober, G.; Keyser, U. F. Biosens. Bioelectron. 2009, 24, 2423-7.

(3) Astier, Y.; Braha, O.; Bayley, H. J. Am. Chem. Soc. 2006, 128, $1705-10$.

(4) Steinbock, L. J.; Lucas, A.; Otto, O.; Keyser, U. F. Electrophoresis 2012, 33, 3480-3487.

(5) Kasianowicz, J. J. J.; Brandin, E.; Branton, D.; Deamer, D. W. W. Proc. Natl. Acad. Sci. U.S.A. 1996, 93, 13770.

(6) Steinbock, L. J.; Otto, O.; Chimerel, C.; Gornall, J.; Keyser, U. F. Nano Lett. 2010, 10, 2493-7.

(7) Shao, Y.; Mirkin, M. J. Am. Chem. Soc. 1997, 119, 8103-8104.

(8) Steinbock, L J; Otto, O.; Skarstam, D R; Jahn, S.; Chimerel, C.; Gornall, J L; Keyser, U. F. J. Phys.: Condens. Matter 2010, 22, 454113.

(9) Gao, C.; Ding, S.; Tan, Q.; Gu, L.-Q. Anal. Chem. 2009, 81, 80-

(10) Kowalczyk, S. W.; Grosberg, A. Y.; Rabin, Y.; Dekker, C. Nanotechnology 2011, 22, 315101.

(11) Storm, A. J.; Chen, J. H.; Ling, X. S.; Zandbergen, H. W.; Dekker, C. Nat. Mater. 2003, 2, 537-40.

(12) Danelon, C.; Santschi, C.; Brugger, J.; Vogel, H. Langmuir 2006, 22, 10711-5.

(13) Chang, H.; Iqbal, S. M.; Stach, E. a.; King, A. H.; Zaluzec, N. J.; Bashir, R. Appl. Phys. Lett. 2006, 88, 103109.

(14) Lo, C. J.; Aref, T.; Bezryadin, A. Nanotechnology 2006, 17, 3264-3267.

(15) Asghar, W.; Ilyas, A.; Billo, J. A.; Iqbal, S. M. Nanoscale Res. Lett. 2011, 6, 372.

(16) Prabhu, A. S.; Freedman, K. J.; Robertson, J. W. F.; Nikolov, Z.; Kasianowicz, J. J.; Kim, M. J. Nanotechnology 2011, 22, 425302.

(17) Radenovic, A.; Trepagnier, E.; Csencsits, R.; Downing, K. H.; Liphardt, J. Appl. Phys. Lett. 2008, 93, 183101.

(18) Zhang, W. M.; Wang, Y. G.; Li, J.; Xue, J. M.; Ji, H.; Ouyang, Q.; Xu, J.; Zhang, Y. Appl. Phys. Lett. 2007, 90, 163102.

(19) Ayub, M.; Ivanov, A.; Hong, J.; Kuhn, P.; Instuli, E.; Edel, J. B.; Albrecht, T. J. Phys.: Condens. Matter 2010, 22, 454128.

(20) Chen, P.; Gu, J.; Brandin, E.; Kim, Y.-R. Y. R.; Wang, Q.; Branton, D. Nano Lett. 2004, 4, 2293-2298.

(21) Kim, Y.-R.; Lee, I.-H.; Min, J.; Kim, A.-G.; Kim, S.; Kim, K.; Namkoong, K.; Ko, C. Biosens. Bioelectron. 2007, 22, 2926-31.

(22) Schibel, A. E. P.; Edwards, T.; Kawano, R.; Lan, W.; White, H. S. Anal. Chem. 2010, 82, 7259-66.

(23) Platt, M.; Willmott, G. R.; Lee, G. U. Small 2012, 8, 2436-44.

(24) Wanunu, M.; Dadosh, T.; Ray, V.; Jin, J.; McReynolds, L.; Drndić, M. Nat. Nanotechnol. 2010, 5, 807-14.

(25) Takahashi, Y.; Shevchuk, A. I.; Novak, P.; Babakinejad, B.; Macpherson, J.; Unwin, P. R.; Shiku, H.; Gorelik, J.; Klenerman, D.; Korchev, Y. E.; Matsue, T. Proc. Natl. Acad. Sci. U.S.A. 2012, 109, $11540-5$.

(26) Gornall, J. L.; Mahendran, K. R.; Pambos, O. J.; Steinbock, L. J.; Otto, O.; Chimerel, C.; Winterhalter, M.; Keyser, U. F. Nano Lett. 2011, 11, 3334-3340.

(27) Hernández-Ainsa, S.; Muus, C.; Bell, N. a W.; Steinbock, L. J.; Thacker, V. V; Keyser, U. F. The Analyst 2012, 16-18.

(28) Steinbock, L. J.; Keyser, U. F. In Methods in Molecular Biology; Gracheva, M. E., Ed.; Springer Science: Totowa, NJ, 2012; Vol. 870, pp 135-145.

(29) Raillon, C.; Cousin, P.; Traversi, F.; Garcia-Cordero, E.; Hernandez, N.; Radenovic, A. Nano Lett. 2012, 12, 1157-64.

(30) Raillon, C.; Granjon, P.; Graf, M.; Steinbock, L. J.; Radenovic, A. Nanoscale 2012, 4, 4916-24.

(31) Willmott, G. R.; Smith, B. G. Nanotechnology 2012, 23, 088001.

(32) Thacker, V. V; Ghosal, S.; Hernández-Ainsa, S.; Bell, N. A. W.; Keyser, U. F. Appl. Phys. Lett. 2012, 101, 223704. 
(33) Li, J.; Gershow, M.; Stein, D.; Brandin, E.; Golovchenko, J. A. Nat. Mater. 2003, 2, 611-615. 